\title{
Traffic Risk Modelling and Analysis under Airport-Like Simulation Environment
}

\author{
Yanting Sheng $\mathbb{D}^{\mathrm{D}},{ }^{1}$ Rui Feng, ${ }^{2}$ and Salvatore Antonio Biancardo $\mathbb{i D}^{3}$ \\ ${ }^{1}$ China Airport Construction Group Northwest Design \& Research Institute Co., Ltd., Xi'an 710075, Shaanxi, China \\ ${ }^{2}$ College of Transportation, Chang'an University, Xi'an 710064, Shaanxi, China \\ ${ }^{3}$ Department of Civil, Construction and Environmental Engineering, Federico II University of Naples, Naples, Italy
}

Correspondence should be addressed to Yanting Sheng; 2019222042@chd.edu.cn

Received 1 October 2021; Revised 28 November 2021; Accepted 7 December 2021; Published 6 January 2022

Academic Editor: Zhenzhou Yuan

Copyright (c) 2022 Yanting Sheng et al. This is an open access article distributed under the Creative Commons Attribution License, which permits unrestricted use, distribution, and reproduction in any medium, provided the original work is properly cited.

Traffic safety plays a crucial role in the development of autonomous vehicles which attracts significant attention in the community. It is a challenge task to ensure autonomous vehicle safety under varied traffic environment interference, especially for airport-like closed-loop conditions. To that aim, we analyze autonomous vehicle safety at typical roadway conditions and traffic state constraints (e.g., car-following state at different speed distributions) by simulating the airport-like traffic conditions. The experimental results suggest that traffic collision risk is in a positive relationship with the speed difference and distance among adjacent vehicles. More specifically, the autonomous vehicle may collide with neighbors when the time to collision (TTC) indicator is lower than $4 \mathrm{~s}$, and vice versa. The research findings can help both research community and practioners obtain additional information for improving traffic safety for autonomous vehicles.

\section{Introduction}

The development of autonomous vehicle is considered as the future tendency due to advantages of safety, low carbon emission, higher commuting efficiency, etc. It is found that automated vehicles can be easily deployed at closed-loop environments (such as airport, terminal, etc.) for the purpose of goods transmission, but the autonomous vehicle encounters various problems which may lead to severe traffic accidents [1]. Previous studies suggested that performance evaluation and verification for autonomous vehicle is significantly different from those of the conventional vehicles [2-4]. More specifically, previous vehicle movement simulations were mainly implemented targeting on specific traffic scenes, and thus the modelling results may fail to obtain satisfied results under different roadway scenes. For instance, autonomous vehicles travel in airport will suffer less interference of traffic violation. In that manner, additional typical traffic scenes are required to better verify the model performance, and thus support autonomous vehicle development.
It is noted that autonomous vehicle driving test phase can be roughly divided into virtual simulation test, private field test, and the roadway travelling test $[5,6]$. Currently, many studies were implemented to verify the autonomous vehicle performance without triggering life loss, roadway intermittence, and property damage [7]. Xing et al. proposed a novel framework to model the car-following vehicle behavior with a comprehensive joint time-series approach [8]. Zhang et al. proposed a connect-and-merge residual network to extract simplified but robust vehicle features under complex roadway scene interference [9]. Ma et al. proposed an efficient lane detection model under varied weather conditions via the help of image classification and hybrid isomeric operator [10]. Similar researches can be found in $[11,12]$.

Previous car-following model verification is mainly implemented on the virtual platform due to strict restrictions to maneuver autonomous vehicle on roadways (traffic regulations, etc.) $[13,14]$. Moreover, it is not easy to implement car-following models in real world considering that we cannot enumerate each traffic scenario. Significant 
attentions were paid to generate virtual transportation sensory data (i.e., typical yet not-easy-accessible traffic dataset). The study aims to develop a novel traffic risk evaluation module for enclosed traffic environments (e.g., airport-like surroundings). More specifically, the study analyzes potential traffic risk under typical airport traffic situations in a simulation manner, and the car-following model performance for different traffic scenarios in the airport-like enclosed loop environment is further visualized in our study.

Our contributions can be summarized as follows: (1) we analyze the pros and cons for traffic collision simulation models on different traffic simulation platforms; (2) we simulate varied traffic scenarios under typical enclosed traffic environment (e.g., airport-like condition); (3) we quantify the traffic simulation module performance under typical traffic scenarios (i.e., different speeds and headway distributions). The remainder of the paper is organized as follows. Section 2 presents the literature survey on the topics of traffic collision simulations, traffic scene establishment, and the simulation platform establishment. Section 3 demonstrates the evaluation criterion for the purpose of model quantitative analysis and Section 4 illustrates the experimental setups. Section 5 analyzes the simulation results and Section 6 briefly concludes the study.

\section{Literature Survey}

2.1. Traffic Collision Simulation Models. Traffic collision simulations provide quantitative results for the purpose of verifying accident prevention model performance. Suh et al. developed a vehicle trajectory planning model, which integrated the lane change probability and deterministic prediction algorithms [15]. The simulation results showed that autonomous vehicle can successfully handle lane change challenges without triggering traffic accident. Li et al. proposed a game-theoretic traffic model for the purpose of evaluating various decision-making and control systems of autonomous vehicles [16]. Quddus et al. proposed a data-driven approach with the help of VISSIM platform to estimate the traffic collision probability [17]. Pan et al. extracted vehicle trajectory information by scanning street scenes with the help of Lidar and camera data [18]. Huang et al. proposed a force-based heterogeneous traffic simulation model to identify dangerous behaviors of various roadway user, which may trigger traffic accident in real world [19]. Similar studies can be found in $[20,21]$.

2.2. Traffic Scene Establishment. It is crucial to collect comprehensive traffic scenes when implementing autonomous vehicle simulation studies. To that aim, Zhu et al. proposed a road scenery prediction model by recognizing roadway boundary points with a convolutional neural network [22]. Cui et al. proposed a multi-modal traffic scene simulation framework by integrating both roadway images and geographic data, which was verified on public-available dataset [23]. Yuan et al. developed a three-dimensional traffic scene construction model, which consists of the scene analysis and scene model construction [24]. Garzón et al. introduced an open source tool to generate varied yet typical traffic scenes for autonomous vehicle under different traffic state conditions, with the support of microscopic, multimodal traffic simulator and a complex 3D simulator [25]. Huo et al. established a three-dimensional traffic scene framework based on semantic segmentation logic [26]. Berumen et al. proposed an abstract simulation scenario generation framework considering both autonomous vehicle safety and computational cost [27]. Similar researches can also be found in $[28,29]$.

2.3. Traffic Simulation Platforms. Simulation platform is crucial for implementing autonomous vehicle traffic safety analysis, and thus significant focuses are paid to develop an efficient traffic simulation platform. Zhao et al. proposed an automatic simulation test evaluation system based on highway autonomous driving algorithms [30]. Sarat et al. designed a simulation platform to verify the vehicle routing model performance, which can be used to tackle vehicle fleet route optimization problem [31]. Saraoglu et al. proposed a novel fault-chain analysis framework to evaluate safety level of autonomous driving system under different magnitudes (i.e., component level, vehicle maneuvering level, and transportation management level) [32]. Cao et al. constructed virtual and visual simulation display platform with the support of web graphics library [33]. Similar researches can be found in [34-36]. Currently, autonomous vehicle test can be implemented at limited locations due to technical and legal restrictions. We cannot implement fatal yet important autonomous vehicle experiments on real-world roadways. The SCANeR studio platform provides sufficient tools and mathematical models, which helps transportation practitioners and scholars easily build virtual traffic scenarios [37]. Thus, the SCANeR studio tool is used to establish traffic safety platform in our study.

In sum, previous studies indicated that traffic collision avoidance relevant studies focused on urban traffic situations. In such condition, the roadway traffic interference randomly originated from pedestrians and aggressive yet unpredictable driving behaviors that cannot be easily modeled. In addition, previous studies may simplify traffic participant behaviors for establishing risky traffic scenes in the traffic simulation platforms. Our study focused on transportation risk evaluation under enclosed traffic conditions, whilst traffic interference was different from the conventional roadways. Moreover, we comprehensively evaluate driving behaviors by analyzing varied car-following states in the enclosed environment such as airport-like roadway condition.

\section{Evaluation Metrics}

3.1. Distance-Based Traffic Risk Evaluation Measurement. For the purpose of avoiding traffic collision, the rear vehicle is supposed to keep a safety distance against the front 
vehicle. Both of the front and rear vehicles keep sufficient braking distance and time to avoid potential accidents under emergency conditions (e.g., sudden acceleration/deceleration). It is noted that minimum safety distance and time can be different according to vehicle speed. We can estimate roadway traffic accident probability by quantifying the relationship between vehicle headway distance and vehicle speed. More specifically, it is quite possible to have a traffic accident when equation (1) is not satisfied, and vice versa.

$$
\frac{v_{i}^{2}}{254\left(f_{z}+i_{z}\right)}+d \geq \frac{v_{j} \tau}{3.6}+\frac{v_{j}^{2}}{254\left(f_{z}+S_{l}\right)}+D+L_{v b},
$$

where $d$ is the headway distance between two neighboring vehicles and $v_{i}$ and $v_{j}$ are speeds for the front and rear vehicles, respectively. The parameter $\tau$ is the overall time overhead consisting of dangerous traffic situation awareness and brake operation implementation. We set default value for $\tau$ to $2 \mathrm{~s}$ in our study. The symbol $f_{z}$ is the longitudinal adhesion coefficient for the road, and the parameter ranges from 0.45 to 0.7 . $S_{l}$ is the longitudinal roadway slope, and $L_{v b}$ is the vehicle length (which is usually shorter than $6 \mathrm{~m}$ ). The parameter $D$ is the safe parking distance, and the default value is set to $2 \mathrm{~m}$ in our study.

\subsection{Time-Based Traffic Risk Evaluation Measurement.} Time to collision (TTC) is another typical yet efficient indicator to measure traffic safety level, which presents carfollowing status between two neighboring vehicles (i.e., the front and rear vehicles). The TTC indicator is defined as the duration of the vehicle collision process when the speed difference remains constant under certain time span $t$ (i.e., the rear vehicle speed is larger than the counterpart of the front vehicle). Generally speaking, we consider that traffic accident is likely to happen when the TTC is shorter than the overhead of driver perception and reaction time. More specifically, the rear vehicle speed at timestamp $t_{1}$ is higher than that of the front vehicle, and thus a traffic collision event is anticipated at time $t_{2}$ without taking further measurements. The time difference between timestamp $t_{1}$ and $t_{2}$ is named as the TTC, which is calculated in equation (2). Previous studies suggested that roadway traffic is safe when the TTC value is larger than $2 \mathrm{~s}[38,39]$. Moreover, we considered the traffic situation is safe when the TTC ranges from $2 \mathrm{~s}$ to $6 \mathrm{~s}$, and we set TTC threshold into $4 \mathrm{~s}$ in our study. More specifically, the traffic condition was considered safe (not safe) when the TTC value was larger than $4 \mathrm{~s}$ (smaller than $2 \mathrm{~s}$ ):

$$
\begin{aligned}
& \text { TTC }=\frac{X_{i-1}(t)-X_{i}(t)-l_{i-1},}{v_{i}(t)-v_{i-1}(t)}, \\
& v_{i}(t)>v_{i-1}(t),
\end{aligned}
$$

where parameter $X_{i-1}(t)$ and $X_{i}(t)$ are the front (i.e., the $(i-1)$ th) and rear (i.e., the $i$ th) vehicle positions at timestamp $t$, respectively. The symbols $v_{i-1}(t)$ and $v_{i}(t)$ are the front and rear vehicle speeds at the timestamp $t$, and $l_{i-1}$ is the front vehicle length.

\section{Experiment Setups}

4.1. Platform. We employ the SCANeR studio software to implement traffic simulation scenarios in our study though many software and platforms are available. The main advantage is that the software provides various but complete components to simulate real-world traffic (especially for the enclosed airport-like environment). Typical models and tools for the SCANeR studio include road environment, vehicle moving state, varied traffic conditions, inductive loop detectors, drivers, traffic lights, various weather conditions, etc. The traffic platform provides different modules to help both researchers and engineers easily customize their simulation scenarios.

4.2. Roadway Design. We integrate both roadway design manual and simulation demand to design potential roadway conditions for autonomous vehicles. For a given intersection, we define the arterial lane in the north-south direction as the first roadway segment with speed limit at $60 \mathrm{~km} / \mathrm{h}$. The lane width is set to $3.5 \mathrm{~m}$, the marginal strip is set to $0.5 \mathrm{~m}$, and the outer separator is set to $1.5 \mathrm{~m}$. Note that the width of the non-motorized road surface is $2.5 \mathrm{~m}$, and the sidewalk is set to $3 \mathrm{~m}$. The separation zone is surrounded by vertical curbs with height around $15 \mathrm{~cm}$. The width of the double white line in the center of the road is configured as $20 \mathrm{~cm}$. The second roadway segment is defined as a branch corridor with speed limit at $40 \mathrm{~km} / \mathrm{h}$, with each lane width at $3.25 \mathrm{~m}$. The marginal strip for the second roadway segment is $0.25 \mathrm{~m}$, and the roadway is separated by the central double yellow line with width of $20 \mathrm{~cm}$. The third roadway segment is a secondary trunk road, which locates east-west bound at the intersection. The separation zone is similar to those of the counterparts of the first and second roadway segments. Note that the maximum speed is $50 \mathrm{~km} / \mathrm{h}$ for the third highway segment, and lane width is $3.5 \mathrm{~m}$. The arterial of the east-west bound is set as the fourth roadway segment, and the road geometry is similar to that of the first road (i.e., design speed is $60 \mathrm{~km} / \mathrm{h}$, lane width at $3.5 \mathrm{~m}$, non-motorized road width of $2.5 \mathrm{~m}$ ). The roadway layout overview is shown in Figure 1.

4.3. Car-Following Scenario. The car-following scene aims to test the safety performance of autonomous vehicle under different car-following states (e.g., stop-and-go, emergency braking function). For a given car-following state, both the front and rear vehicles travel in the same lane, and the two vehicles are initialized with same kinematic information (i.e., speed, travelling direction). We consider the front vehicle as the interference vehicle, and thus the vehicle travelling status variation affects the rear vehicle travelling state. The specific simulation plan is shown as follows: we select the first arterial road in the north-south direction, and the design speed is set to $60 \mathrm{~km} / \mathrm{h}$. The width of the motor vehicle lane is assigned to $3.5 \mathrm{~m}$, the marginal strip is $0.5 \mathrm{~m}$, the outer separator is $1.5 \mathrm{~m}$, the width of the non-motorized road surface is $2.5 \mathrm{~m}$ and the sidewalk is $3 \mathrm{~m}$. The speed limit on roads is $60 \mathrm{~km} / \mathrm{h}$. The start acceleration and stop 


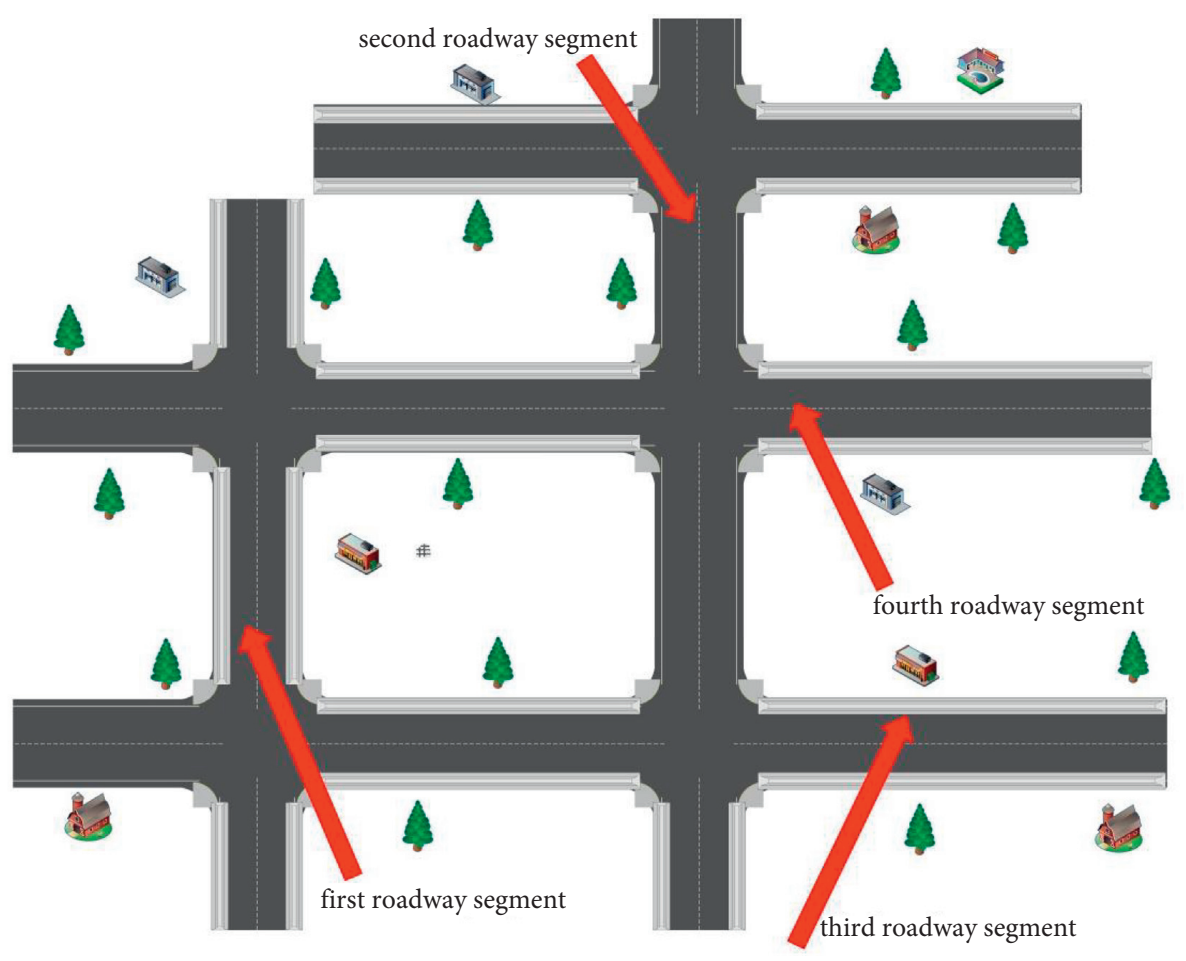

FIgURE 1: Roadway layout overview.

deceleration of the interference vehicle refer to the typical start acceleration and emergency stop deceleration, which are $2.5 \mathrm{~m} / \mathrm{s}^{2}$ and $-2 \mathrm{~m} / \mathrm{s}^{2}$, respectively.

It is believed that driver will not collide with the obstacle in any form from the moment the obstacle ahead the vehicle is discovered till the end of the vehicle braking procedure. The distance in this process is called the minimum safety distance. From the perspective of vehicle braking process, the relationship between vehicle braking distance and vehicle braking initial speed can be exploited with the least square method. Following the vehicle safety distance manual, the safety distance should not be smaller than 50 meters when vehicle speed is around $50 \mathrm{~km} / \mathrm{h}$. In automated vehicle era, a large amount of sensors (such as millimeter wave radar, multi-threaded LiDAR, etc.) are deployed to vehicles to enhance capability of accurate vehicle environment perception. Currently, many studies indicate that the newly emerging communication techniques (such as $5 \mathrm{G}$ technique) are capable of transmitting data with low time delay $[40,41]$.

It is similar to implement the experimental scenarios when we set speeds for both the front and rear vehicles to the maximum values. In practice, the autonomous vehicle combines the road speed limit and traffic conditions to select the speed. We set the maximum speed of the main vehicle and the interference vehicle to be the same to maintain the car-following state. The speed and initial distance of the main vehicle and the interference vehicle are shown in Table 1. The specific verification scenarios are as follows: (1) the initial distance between the main vehicle and the interference vehicle is $40 \mathrm{~m}$, and the main vehicle and interference vehicle are set at different maximum speeds; (2) the maximum speed of the main vehicle and the interference vehicle are both $60 \mathrm{~km} / \mathrm{h}$, and the car-following with initial distance is set to $30 \mathrm{~m}, 40 \mathrm{~m}$, and $50 \mathrm{~m}$. Table 2 demonstrates speed and initial distance distributions of the two vehicles. The experimental setting considers the factors of simulation experiment, and factors of vehicle speed, acceleration, distance between main vehicle, and interference vehicle are considered in the study.

\section{Results}

\subsection{Traffic Analysis via Various Maximum Speed Distributions}

5.1.1. Car-Following Analysis at Different Maximum Speed Constraints. In the car-following scenario, the initial distance between the main vehicle and the interference vehicle is the same. The simulation results of the car-following scene at different maximum speeds are analyzed as follows. (1) In the car-following scene, the initial distance is set to $40 \mathrm{~m}$ and the maximum speeds of both vehicles are set to $40 \mathrm{~km} / \mathrm{h}$. The main vehicle accelerates from $0 \mathrm{~km} / \mathrm{h}$ to the maximum speed (i.e., $40 \mathrm{~km} / \mathrm{h}$ ). When the interference vehicle decelerates, the main vehicle intends to overtake the vehicle by change its travelling lane to the left side. But, the vehicle decelerates and continues to follow the front vehicle by accelerating to the maximum speed of $40 \mathrm{~km} / \mathrm{h}$ when the interference vehicle accelerates its moving speed. (2) In the car-following scene with an initial distance of $40 \mathrm{~m}$ and the maximum speed of both vehicles travelling at $50 \mathrm{~km} / \mathrm{h}$, the simulation results are illustrated as follows. The main vehicle accelerates from $0 \mathrm{~km} / \mathrm{h}$ to the maximum speed of $50 \mathrm{~km} / \mathrm{h}$. When the 
TABLE 1: Varied speeds and same initial distance of main vehicle and interference vehicle.

\begin{tabular}{|c|c|c|c|}
\hline \multicolumn{2}{|c|}{ Vehicle } & Speed & Initial distance $(\mathrm{m})$ \\
\hline Main vehicle & Interference vehicle & $40 \mathrm{~km} / \mathrm{h}$ & 40 \\
\hline Main vehicle & Interference vehicle & $50 \mathrm{~km} / \mathrm{h}$ & 40 \\
\hline Main vehicle & Interference vehicle & $60 \mathrm{~km} / \mathrm{h}$ & 40 \\
\hline
\end{tabular}

TABLE 2: Same speed and different initial distances for main vehicle and interference vehicle.

\begin{tabular}{|c|c|c|c|}
\hline \multicolumn{2}{|c|}{ Vehicle } & Speed & Initial distance $(\mathrm{m})$ \\
\hline Main vehicle & Interference vehicle & $60 \mathrm{~km} / \mathrm{h}$ & 30 \\
\hline Main vehicle & Interference vehicle & $60 \mathrm{~km} / \mathrm{h}$ & 20 \\
\hline Main vehicle & Interference vehicle & $60 \mathrm{~km} / \mathrm{h}$ & 10 \\
\hline
\end{tabular}

interference vehicle decelerates, the main vehicle has the intention of overtaking behavior. The vehicle decelerates and continues to follow in the manner of accelerating to the maximum speed of $50 \mathrm{~km} / \mathrm{h}$ when the interference vehicle accelerates its speed. (3) In the third car-following scene, the initial distance is set to $40 \mathrm{~m}$ and the maximum speeds of both vehicles are setting at $60 \mathrm{~km} / \mathrm{h}$. The main vehicle accelerates from $0 \mathrm{~km} / \mathrm{h}$ to a maximum speed of $60 \mathrm{~km} / \mathrm{h}$, which is similar to those of the previous two traffic scenarios. Moreover, the main vehicle wants to overtake the interference vehicle when it travels at a constant speed. Besides, the main vehicle decelerates its speed when the interference vehicle accelerates. In that way, the main vehicle decelerates and continues to follow the interference counterpart by accelerating to the maximum speed of $60 \mathrm{~km} / \mathrm{h}$.

The speed curve distributions of the main vehicle and interference in the three cases are shown in Figures 2 and 3, respectively. Note that the maximum speeds for the main and interference vehicles are same without further specifications in the study. The overall perception, planning and control response of the main vehicle are supposed to be the same for different speeds during the car-following procedure. The acceleration curves of the main vehicle in the three cases are shown in Figure 4 . It can be seen from the acceleration curves that after the interference vehicle decelerates, the main vehicle first has a short deceleration process. Then, the main vehicle is ready to accelerate and overtake the interference vehicle. After the interference vehicle accelerates, the main vehicle decelerates and continues to follow the front vehicle.

The distance difference curves in the three cases are shown in Figure 5. When the initial distance is set to $40 \mathrm{~m}$, the distance between the main vehicle and the interference vehicle shows an increasing tendency when the vehicle accelerates in the simulation procedure. We further analyze the car-following procedure from the perspective of traffic environment perception. The initial distance and maximum speed are set to same values in the three car-following procedures. Besides, the main vehicle recognizes the behavior of the front vehicle by identifying the vehicle kinematic information (i.e., speed, acceleration, deceleration), and further vehicle maneuvering decisions are made to enhance traffic safety by following or overtaking the interference vehicle.
5.1.2. Traffic Risk Analysis at Different Maximum Speed Constraints. In the car-following scene with the same initial distance, the indicators for evaluating the safety of the autonomous vehicle are used for evaluation. The initial distance is selected as $40 \mathrm{~m}$, and the maximum speed of the main vehicle and the interference vehicle is $40 \mathrm{~km} / \mathrm{h}, 50 \mathrm{~km} /$ $\mathrm{h}$, and $60 \mathrm{~km} / \mathrm{h}$. The evaluation results of the two indicators are demonstrated as follows. We employ the distance-based risk evaluation indicator to evaluate the risk of autonomous vehicles. According to the requirements of the scene, the risk analysis of the main vehicle and the interference vehicle with the same initial distance and different speed is carried out, and the three groups of traffic situations are analyzed in detail as follows: (1) $v_{i}$ is set to $40 \mathrm{~km} / \mathrm{h}$ and $v_{j}$ set to $40 \mathrm{~km} / \mathrm{h}$; the factors $f_{z}$ and $f_{z}$ are set to 0.7 and 0.06 (i.e., maximum values). Besides, the parameter values for $d, \tau, D$ and $L_{v b}$ are set to $45 \mathrm{~m}, 2.5 \mathrm{~s}, 2 \mathrm{~m}$ and $5 \mathrm{~m}$; (2) both of $v_{i}$ and $v_{j}$ are set to $50 \mathrm{~km} / \mathrm{h}$, and $f_{z}, S_{l}, d, \tau, D$ and $L_{v b}$ are assigned with 0.7 , $0.06,45 \mathrm{~m}, 2.5 \mathrm{~s}, 2 \mathrm{~m}$ and $5 \mathrm{~m}$; (3) the parameters for $v_{i}, v_{j}$, $f_{z}, S_{l}, d, \tau, D$ and $L_{v b}$ are set to $60 \mathrm{~km} / \mathrm{h}, 60 \mathrm{~km} / \mathrm{h}, 0.7,0.06$, $45 \mathrm{~m}, 2.5 \mathrm{~s}, 2 \mathrm{~m}$ and $5 \mathrm{~m}$. The simulation results indicate that there is no traffic collision risk between the main vehicle and the interference vehicle under the three traffic scenarios. The distance-based risk evaluation indicators suggest that vehicles can safely travel in the current traffic situations. In other words, there is no collision risk between the main and the interference vehicles with the provided vehicle speed and distance values.

We employ the time-based risk evaluation indicator TTC to evaluate the risk of autonomous vehicles. According to the simulation experiment results, the maximum speeds of the main vehicle and the interference vehicle are $40 \mathrm{~km} / \mathrm{h}$, $50 \mathrm{~km} / \mathrm{h}$, and $60 \mathrm{~km} / \mathrm{h}$ and the initial distance to the interference vehicle is $40 \mathrm{~m}$. The two-vehicle distance diagram between the main vehicle and the interference vehicle is shown in Figure 6. It is observed that the two vehicles were quite close to each other (approximately $5 \mathrm{~m}$ ) when the maximum speed was set to $40 \mathrm{~km} / \mathrm{h}$ and the time duration for car-following state lasts over $60 \mathrm{~s}$. It is observed that the TTC value may show unrealistic oscillations when the maximum speed is $40 \mathrm{~km} / \mathrm{h}$. The main reason was that reaction time for drivers is lower than those counterparts with speeds at 50 and $60 \mathrm{~km} / \mathrm{h}$, respectively. In another word, the aggressive drivers may keep smaller distance away from the 


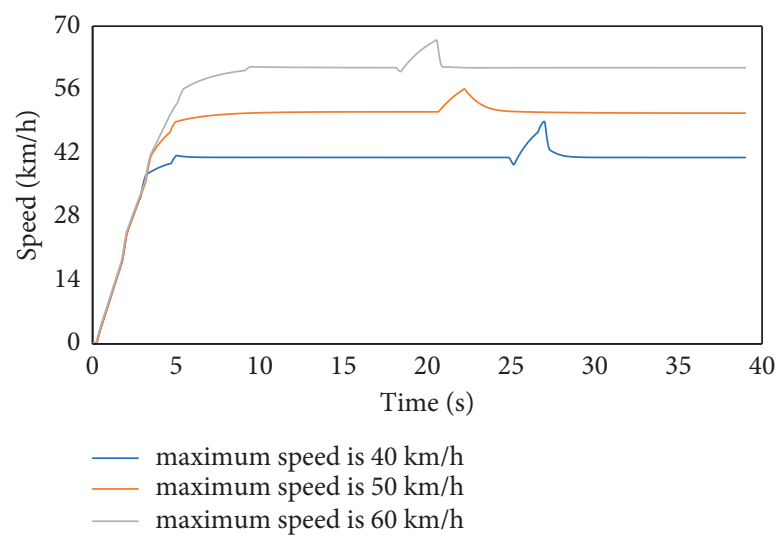

FIgURE 2: Maximum speed distributions of main vehicle.

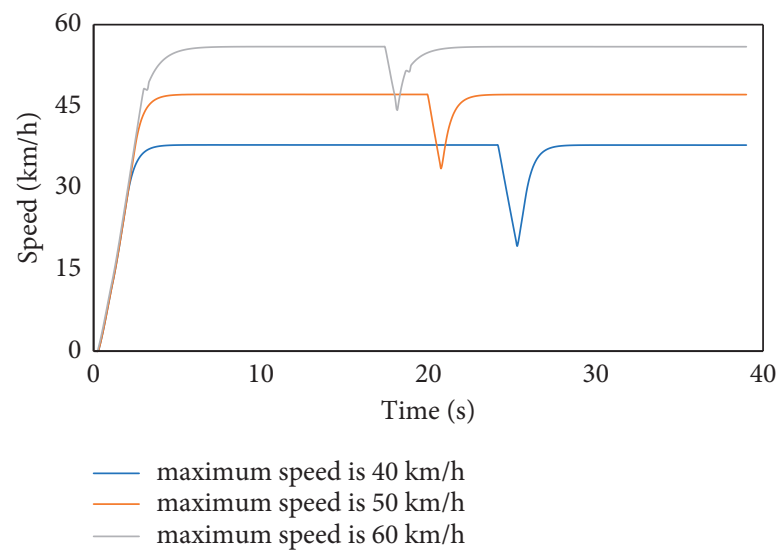

Figure 3: Maximum speed distributions of interference vehicle.

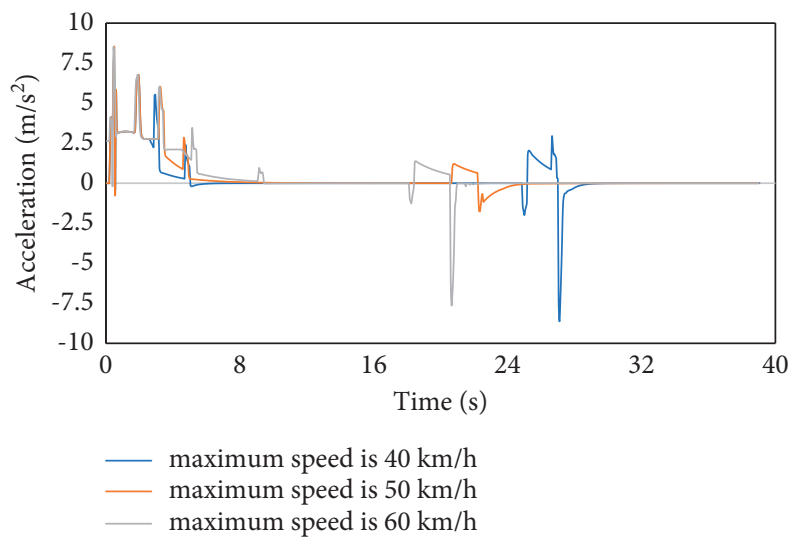

FIgURE 4: Acceleration curve distributions of the main vehicle (note that negative acceleration indicates deceleration).

front vehicle, and the mild drivers may travel with a larger headway distance.

Based on the above-mentioned simulation results, we select the situation where the speed of the rear vehicle is greater than the speed of the front vehicle during the carfollowing process. The TTC of the main vehicle is measured as shown in Figure 7. The time-based autonomous driving risk judgment condition is that if the TTC of the main vehicle relative to the interference vehicle is greater than $4 \mathrm{~s}$, the main vehicle is considered in a safety state. More specifically, the minimum TTC value in real world is approximately $3.22 \mathrm{~s}$ when the maximum speed for the main vehicle is set to $50 \mathrm{~km} / \mathrm{h}$. The car-following simulation results indicated that TTC was larger than $3 \mathrm{~s}$ when the 


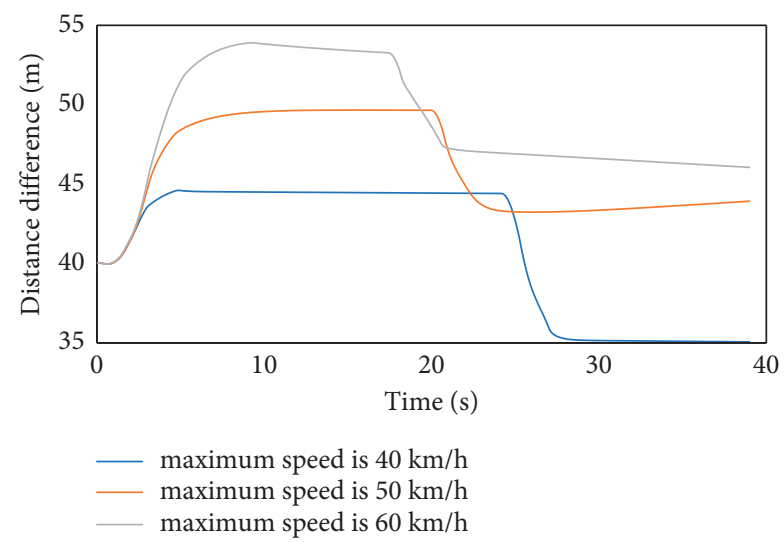

Figure 5: The distance difference between the main vehicle and the interference vehicle.

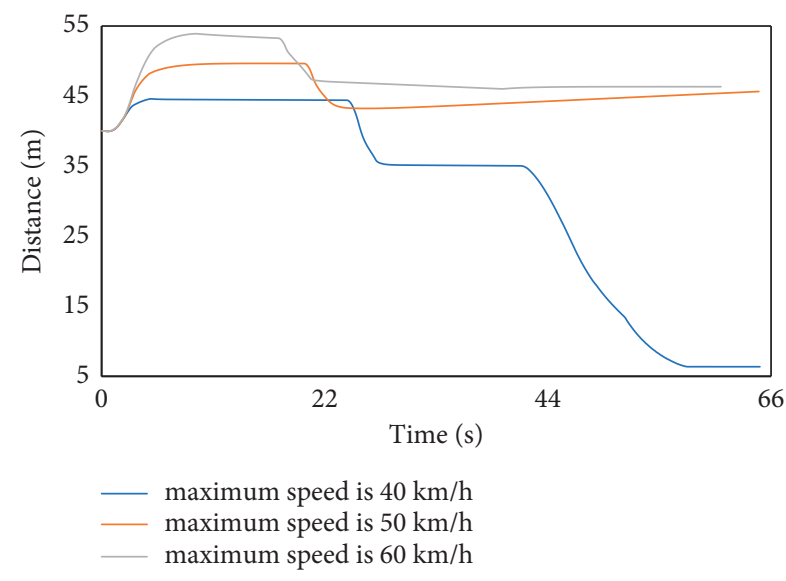

Figure 6: The distance between the front and rear vehicles.

maximum speed reached $50 \mathrm{~km} / \mathrm{h}$. Besides, the TTC value was smaller than $4 \mathrm{~s}$ under the traffic state (i.e., the maximum speed was set to $50 \mathrm{~km} / \mathrm{h}$ ). It can be inferred that TTC setting can be narrowed down with varied speed limitations and roadway conditions. In sum, when the initial distance of the main vehicle and the interference vehicle is $40 \mathrm{~m}$ and the maximum speed is $40 \mathrm{~km} / \mathrm{h}, 50 \mathrm{~km} / \mathrm{h}$, and $60 \mathrm{~km} / \mathrm{h}$, respectively, the risk assessment indicators based on distance and time were introduced to evaluate the risk of autonomous vehicle for the car-following state. The distance-based risk evaluation indicator can successfully simulate the transportation risk in the real-world applications. Moreover, the time-based risk assessment indicator may fail to comprehensively evaluate the model performance when the relative speed of the two vehicles is too small and the relative distance is too large.

\subsection{Traffic Analysis via Various Space Headway Distributions}

5.2.1. Car-Following Analysis at Different Space Headways. In the car-following scene on roads, the simulation results of the car-following scene with the same maximum speed and different initial distance between the main vehicle and the interference vehicle are provided as follows. (1) In the scenario with an initial distance of $30 \mathrm{~m}$, the main vehicle accelerates from $0 \mathrm{~km} / \mathrm{h}$ to a maximum speed of $60 \mathrm{~km} / \mathrm{h}$. When the interference vehicle decelerates, there is a brake operation implemented by the main vehicle. After the interference vehicle accelerates, it decelerates and continues to follow, and then accelerates to the maximum speed of $60 \mathrm{~km} /$ h. (2) In the scenario with an initial distance of $20 \mathrm{~m}$, the main vehicle accelerates from 0 to a maximum speed of $60 \mathrm{~km} / \mathrm{h}$. When the interference vehicle decelerates, there is a brake operation and then there is an intention to overtake the interference vehicle. After the interference vehicle accelerates its speed, the main vehicle decelerates and continues to follow the front vehicle by accelerating to the maximum speed (i.e., $60 \mathrm{~km} / \mathrm{h}$ ). (3) In the scenario with an initial distance of $10 \mathrm{~m}$, the main vehicle changes lanes to the left lane at the beginning of car-following stage. Moreover, the main vehicle accelerates from $0 \mathrm{~km} / \mathrm{h}$ to the maximum speed of $60 \mathrm{~km} / \mathrm{h}$ by following the interference vehicle in the adjacent lanes and achieves overtaking behavior under the condition of interference vehicle decelerating its speed. The distance difference curves between the main vehicle and the interference vehicle under the three conditions are shown in Figure 8. From the distance difference curves between the main vehicle and the interference vehicle, it can be seen that different initial distances make the main vehicle's decision different. The main vehicle makes the decision of car-following when the initial distances are set to $30 \mathrm{~m}$ and $20 \mathrm{~m}$, but, when the initial distance is set to $10 \mathrm{~m}$, the main vehicle conducts lane-change behavior when the interference vehicle slows down.

\subsubsection{Traffic Risk Analysis at Different Space Headway} Constraints. The car-following scene parameters of the main vehicle and the interference vehicle share with the same maximum speed and different initial distances. More specifically, the speeds of the main vehicle and the interference vehicle are both $60 \mathrm{~km} / \mathrm{h}$, and the initial distance is $30 \mathrm{~m}, 20 \mathrm{~m}$, and $10 \mathrm{~m}$. In the car-following scene where the maximum speed of the main vehicle and the interference vehicle are both $60 \mathrm{~km} / \mathrm{h}$, two evaluation indicators are used to evaluate the car-following safety for the main vehicle. More detailed explanations are illustrated as follows: (1) the 


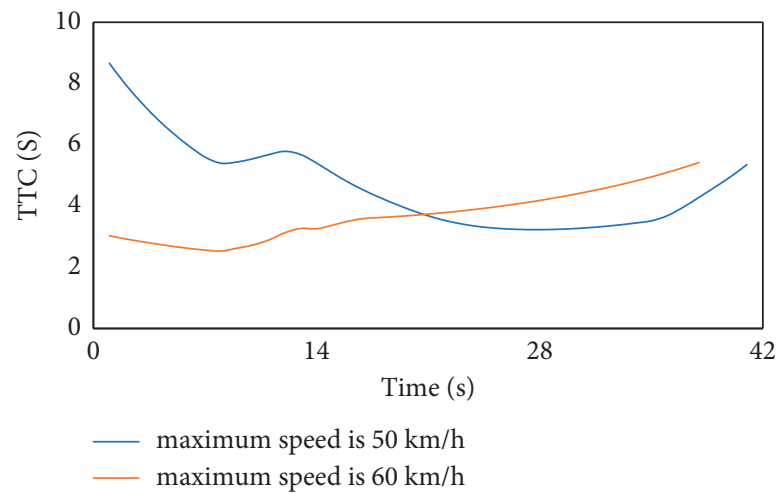

Figure 7: The TTC distributions at different maximum speeds.

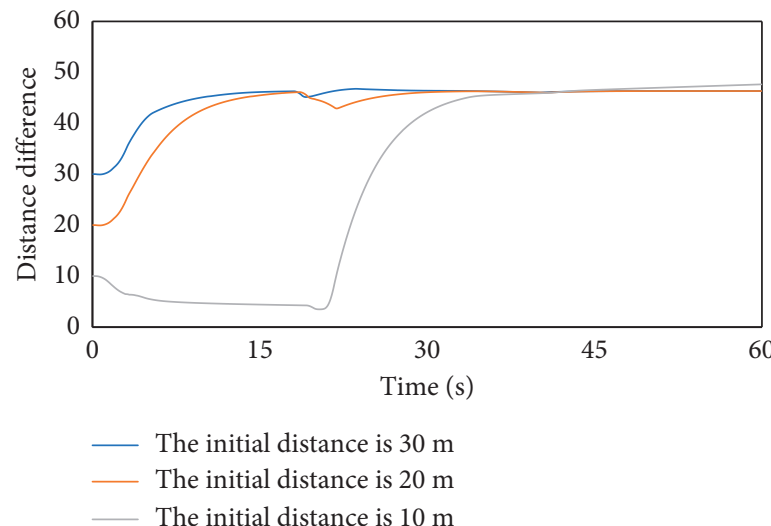

FIGURE 8: The distance difference between the main vehicle and the interference vehicle.

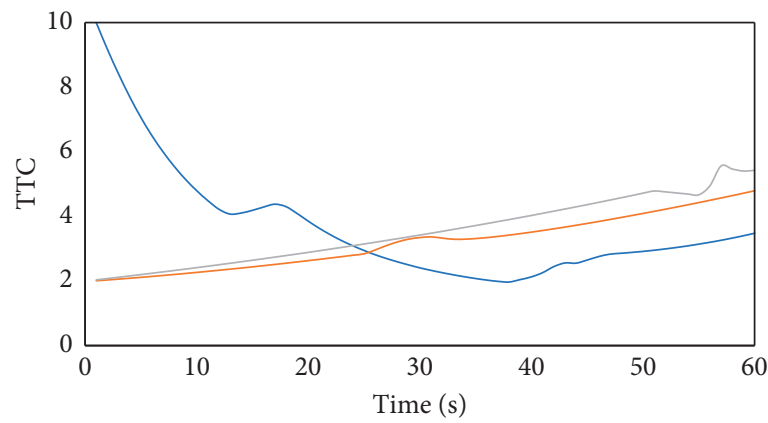

The initial distance is $20 \mathrm{~m}$

Figure 9: The TTC distributions under different initial distances.

distance-based risk evaluation indicator reveals that the main vehicle safety is not relied with the initial distance. The simulation results for traffic safety situation of the two vehicles are similar to those we have analyzed in the above sections. (2) The risk evaluation of main vehicle based on time indicator TTC is demonstrated as follows (see TTC curve distributions in Figure 9). The time-based risk assessment indicator and evaluation models indicated that the traffic state was in safety status when the TTC value was larger than $4 \mathrm{~s}$. We observed that the TTC may be temporarily lower than $2 \mathrm{~s}$ when the maximum vehicle speed reached $60 \mathrm{~km} / \mathrm{h}$. The situation indicated that the overall carfollowing procedure was still quite safe, and reaction time left for drivers under emergency traffic events may be shorter than that of the maximum speed lower than $60 \mathrm{~km} / \mathrm{h}$. Through the risk evaluation indicators based on distance and time, the car-following scenes with different initial distance and same maximum speed are evaluated. The experimental 
results indicated that the evaluation indicators can obtain accurate traffic safety analysis results.

\section{Conclusions}

Autonomous vehicle safety attracts many focuses from varied transportation safety community (airport, maritime field, etc.), and we analyzed vehicle safety under car-following situations at various speed and space headway constraints. More specifically, we designed and modeled the typical car-following scenarios at autonomous vehicle era for an enclosed traffic environment (e.g., airport-like condition). We analyzed the traffic collision risk for the autonomous vehicle in detail, which suggested that the autonomous vehicle is quite safe when the TTC indicator was larger than $4 \mathrm{~s}$. The following directions can be further expanded to enhance our study. First, we focused on autonomous vehicle safety analysis under car-following state, and thus testifying and verifying model performance at more comprehensive traffic states deserve our further endeavor. Second, we can integrate both simulation and real-world traffic data for the purpose of identifying intrinsic traffic factors which impose obvious negative effect on the traffic safety. Last but not least, we can analyze traffic safety under mixed traffic conditions involved with various traffic participants (e.g., autonomous vehicle, trucks, and pedestrians).

\section{Data Availability}

The data are available upon request to the first author through e-mail.

\section{Conflicts of Interest}

The authors declare no conflicts of interest.

\section{Acknowledgments}

The work was jointly supported by the National Natural Science Foundation of China (51978069) and Key Research and Development Plan of Shaanxi Province (2021KWZ-09 and 2021GY-184).

\section{References}

[1] S. Halder, A. Ghosal, and M. Conti, "Secure over-the-air software updates in connected vehicles: a survey," Computer Networks, vol. 178, Article ID 107343, 2020.

[2] J. Jang, J. Ko, J. Park, C. Oh, and S. Kim, "Identification of safety benefits by inter-vehicle crash risk analysis using connected vehicle systems data on Korean freeways," Accident Analysis \& Prevention, vol. 144, Article ID 105675, 2020.

[3] Y. Yang, F. Ma, J. Wang et al., "Cooperative ecological cruising using hierarchical control strategy with optimal sustainable performance for connected automated vehicles on varying road conditions," Journal of Cleaner Production, vol. 275, Article ID 123056, 2020.

[4] Z. Zhang, Y. Cao, M. Ding, L. Zhuang, and J. Tao, "Monocular vision based obstacle avoidance trajectory planning for Unmanned Aerial Vehicle," Aerospace Science and Technology, vol. 106, Article ID 106199, 2020.
[5] T. Rueckelt, I. Stavrakakis, T. Meuser, I. H. Brahmi, D. Böhnstedt, and R. Steinmetz, "Data transmission plan adaptation complementing strategic time-network selection for connected vehicles," Ad Hoc Networks, vol. 82, pp. 146154, 2019.

[6] X. Zhou, Y. Fang, and Y. Mu, "Learning single-shot vehicle orientation estimation from large-scale street panoramas," Neurocomputing, vol. 367, pp. 319-327, 2019.

[7] K. Xie, K. Ozbay, H. Yang, and C. Li, "Mining automatically extracted vehicle trajectory data for proactive safety analytics," Transportation Research Part C: Emerging Technologies, vol. 106, pp. 61-72, 2019.

[8] Y. Xing, C. Lv, and D. Cao, "Personalized vehicle trajectory prediction based on joint time-series modeling for connected vehicles," IEEE Transactions on Vehicular Technology, vol. 69, no. 2, pp. 1341-1352, 2020.

[9] F. Zhang, F. Yang, C. Li, and G. Yuan, "CMNet: a connectand-merge convolutional neural network for fast vehicle detection in urban traffic surveillance," IEEE Access, vol. 7, pp. 72660-72671, 2019.

[10] N. Ma, G. Pang, X. Shi, and Y. Zhai, "An all-weather lane detection system based on simulation interaction platform," IEEE Access, vol. 8, pp. 46121-46130, 2020.

[11] Y. Zhang, G. Zhang, R. Fierro, and Y. Yang, "Force-driven traffic simulation for a future connected autonomous vehicleenabled smart transportation system," IEEE Transactions on Intelligent Transportation Systems, vol. 19, no. 7, pp. 22212233, 2018.

[12] M. Eisenbarth, M. Wegener, R. Scheer et al., "Toward smart vehicle-to-everything-connected powertrains: driving real component test benches in a fully interactive virtual smart city," IEEE Vehicular Technology Magazine, vol. 16, no. 1, pp. 75-82, 2021.

[13] X. Chen, S. Wu, C. Shi et al., "Sensing data supported traffic flow prediction via denoising schemes and ann: a comparison," IEEE Sensors Journal, vol. 20, no. 23, pp. 14317-14328, 2020.

[14] X. Chen, H. Chen, Y. Yang et al., "Traffic flow prediction by an ensemble framework with data denoising and deep learning model," Physica A: Statistical Mechanics and Its Applications, vol. 565, Article ID 125574, 2021.

[15] J. Suh, H. Chae, and K. Yi, "Stochastic model-predictive control for lane change decision of automated driving vehicles," IEEE Transactions on Vehicular Technology, vol. 67, no. 6, pp. 4771-4782, 2018.

[16] N. Li, D. W. Oyler, M. Zhang, Y. Yildiz, I. Kolmanovsky, and A. R. Girard, "Game theoretic modeling of driver and vehicle interactions for verification and validation of autonomous vehicle control systems," IEEE Transactions on Control Systems Technology, vol. 26, no. 5, pp. 1782-1797, 2018.

[17] C. Katrakazas, M. Quddus, and W. H. Chen, "A simulation study of predicting real-time conflict-prone traffic conditions," IEEE Transactions on Intelligent Transportation Systems, vol. 19, pp. 3196-3207, 2019.

[18] C. Chen, X. Zhao, H. Liu, G. Ren, and X. J. Liu, "Influence of adverse weather on drivers' perceived risk during car following based on driving simulations," Journal of Modern Transportation, vol. 27, pp. 282-292, 2019.

[19] Q. Chao, X. Jin, H.-W. Huang, S. Foong, L.-F. Yu, and S.-K. Yeung, "Force-based heterogeneous traffic simulation for autonomous vehicle testing," in Proceedings of the 2019 International Conference on Robotics and Automation (ICRA), pp. 8298-8304, Montreal, QC, Canada, May 2019. 
[20] C. C. Li, C. E. Lin, C. F. Tsai, and S. C. Chiang, "Airborne collision avoidance system for low attitude flights using radio data system," IEEE Aerospace and Electronic Systems Magazine, vol. 25, no. 4, pp. 25-29, 2010.

[21] Y. Xiang, S. Huang, M. Li, J. Li, and W. Wang, "Rear-end collision avoidance-based on multi-channel detection," IEEE Transactions on Intelligent Transportation Systems, vol. 21, no. 8, pp. 3525-3535, 2020.

[22] C. Zhu, Y. Li, Y. Liu et al., "Road scene layout reconstruction based on CNN and its application in traffic simulation," in Proceedings of the 2019 IEEE Intelligent Vehicles Symposium (IV), Paris, France, June 2019.

[23] Z. Cui, Y. Liu, F. Ren, and Q. Zhang, "Multi-model traffic scene simulation with road image sequences and GIS information," in Proceedings of the 2018 IEEE Intelligent Vehicles Symposium (IV), pp. 1943-1948, Changshu, China, June 2018.

[24] J. Yuan, Y. Li, H. Pan, Z. Cui, and Y. Liu, "3D traffic scenes construction and simulation based on scene stages," in Proceedings of the 2018 Chinese Automation Congress (CAC), Xi'an, China, December 2018.

[25] M. Garzón and A. Spalanzani, "An hybrid simulation tool for autonomous cars in very high traffic scenarios," in Proceedings of the 2018 15th International Conference on Control, Automation, Robotics and Vision (ICARCV), pp. 803-808, Singapore, November 2018.

[26] H. Huo, Y. Li, C. Wu, X. Wu, Z. Tian, and Y. Liu, "Semantic segmentation and scene reconstruction for traffic simulation using CNN," in 2019 2nd China Symposium on Cognitive Computing and Hybrid Intelligence (CCHI), Xi'an, China, September 2019.

[27] C. Medrano-Berumen and M. I. Akbaş, "Abstract simulation scenario generation for autonomous vehicle verification," in Proceedings of the 2019 SoutheastCon, pp. 1-6, Huntsville, AL, USA, April 2019.

[28] X. Chen, L. Peng, M. Zhang, and W. Li, "A public traffic demand forecast method based on computational experiments," IEEE Transactions on Intelligent Transportation Systems, vol. 18, no. 4, pp. 984-995, 2017.

[29] J. Wang, W. Lv, Y. Jiang, S. Qin, and J. Li, “A multi-agent based cellular automata model for intersection traffic control simulation," Physica A: Statistical Mechanics and Its Applications, vol. 584, Article ID 126356, 2021.

[30] S. Zhao, Y. Zhai, L. Zhang, C. Chen, and Y. Li, "Simulation test and evaluation system based on HighWay autopilot," in Proceedings of the 2018 IEEE 9th International Conference on Software Engineering and Service Science (ICSESS), Beijing, China, November 2018.

[31] S. C. agavarapu, T. Tripathy, and J. Dauwels, "Development of a simulation platform to implement vehicle routing algorithms for large scale fleet management systems," in Proceedings of the 2017 IEEE 20th International Conference on Intelligent Transportation Systems (ITSC), pp. 256-261, Yokohama, Japan, October 2017.

[32] M. Saraoglu, A. Morozov, and K. Janschek, "MOBATSim: MOdel-based autonomous traffic simulation framework for Fault-Error-Failure chain analysis," IFAC-PapersOnLine, vol. 52, no. 8, pp. 239-244, 2019.

[33] H. Cao, X. Hou, and Y. Feng, "Webgl-based research on virtual visualization simulation display platform of ship," in Proceedings of the 2018 2nd IEEE Advanced Information Management, Communicates, Electronic and Automation Control Conference (IMCEC), pp. 895-899, Xi'an, China, May 2018.
[34] Y. Fu and K. Sun, "Adaptability simulation and analysis of scene matching algorithm," in Proceedings of the 2018 2nd IEEE Advanced Information Management, Communicates, Electronic and Automation Control Conference (IMCEC), pp. 2562-2566, Xi'an, China, May 2018.

[35] S. Chen, Y. Chen, S. Zhang, and N. Zheng, "A novel integrated simulation and testing platform for self-driving cars with hardware in the loop," IEEE Transactions on Intelligent Vehicles, vol. 4, no. 3, pp. 425-436, 2019.

[36] T. Wei, Y. Ming, Lv Zheming et al., "MicroIV: a cooperative driving hardware simulation platform for cooperative-ITS," IEEE Transactions on Vehicular Technology, vol. 67, no. 10, 2018.

[37] M. N. Rastgoo, B. Nakisa, F. Maire, A. Rakotonirainy, and V. Chandran, "Automatic driver stress level classification using multimodal deep learning," Expert Systems with Applications, vol. 138, Article ID 112793, 2019.

[38] Z. Zhang, Y. Huang, and F. Ren, "Driver reaction time in following vehicle at acceleration regime," Journal of Beijing University of Technology, vol. 35, pp. 1220-1124, 2009.

[39] X. Meng, H. Xu, H. Wang, and H. Yao, "Rear-end conflict of freeway work zone based on TTC and DRAC," Journal of Transport Information and Safety, vol. 30, pp. 6-10, 2012.

[40] X. Huang, C. Cao, X. Fan, and Q. Chen, "User assisted dynamic RAN notification area configuration scheme based on delay sensitivity for 5G inactive UEs," IEEE Transactions on Vehicular Technology, vol. 69, no. 3, pp. 2671-2683, 2020.

[41] S. Rostami, S. Lagen, M. Costa, M. Valkama, and P. Dini, "Wake-up radio based access in $5 \mathrm{G}$ under delay constraints: modeling and optimization," IEEE Transactions on Communications, vol. 68, no. 2, pp. 1044-1057, 2020. 